\title{
The X-/Gamma-ray camera ECLAIRs for the Gammay-ray burst mission SVOM
}

\author{
Godet O. ${ }^{a, b}$, Nasser G. ${ }^{a, b}$, Atteia J.-L. ${ }^{a, b}$, Cordier B. ${ }^{c}$, Mandrou P. ${ }^{a, b}$, Barret D. ${ }^{a, b}$, Triou H. ${ }^{c}$, \\ Pons R. ${ }^{a, b}$, Amoros C. ${ }^{a, b}$, Bordon S. ${ }^{a, b}$, Gevin O. ${ }^{c}$, Gonzalez F. ${ }^{e}$, Götz, D. ${ }^{c}$, A. Gros ${ }^{c}$, Houret \\ B. ${ }^{a, b}$, Lachaud C. ${ }^{d}$, Lacombe K. ${ }^{a, b}$, Marty W. ${ }^{a, b}$, Mercier K. ${ }^{e}$, Rambaud D. ${ }^{a, b}$, Ramon P. ${ }^{a, b}$, \\ Rouaix G. ${ }^{a, b}$, Schanne S. ${ }^{c}$, Waegebaert V. ${ }^{a, b}$ \\ ${ }^{a}$ Université de Toulouse, OMP-UPS, Institut de Recherche en Astrophysique et Planétologie \\ (IRAP), Toulouse, France; \\ ${ }^{b}$ CNRS, IRAP, 9 avenue du colonel Roche, 31028 Toulouse Cedex 4, France; \\ ${ }^{c}$ CEA Saclay, DSM/IRFU/service d'Astrophysique, 91191, Gif-sur-Yvette, France; \\ ${ }^{d}$ AstroParticule et Cosmologie (APC), CNRS-UMR 7164, Universit de Paris 7 Denis Diderot, \\ 75205, Paris, France; \\ ${ }^{e}$ CNES, 18 Av. Edouard Belin, 31401 Toulouse cedex 9, France;
}

\begin{abstract}
We present ECLAIRs, the Gamma-ray burst (GRB) trigger camera to fly on-board the Chinese-French mission SVOM. ECLAIRs is a wide-field $(\sim 2 \mathrm{sr})$ coded mask camera with a mask transparency of $40 \%$ and a $1024 \mathrm{~cm}^{2}$ detection plane coupled to a data processing unit, so-called UGTS, which is in charge of locating GRBs in near real time thanks to image and rate triggers. We present the instrument science requirements and how the design of ECLAIRs has been optimized to increase its sensitivity to high-redshift GRBs and low-luminosity GRBs in the local Universe, by having a low-energy threshold of $4 \mathrm{keV}$. The total spectral coverage ranges from 4 to 150 $\mathrm{keV}$. ECLAIRs is expected to detect $\sim 200 \mathrm{GRBs}$ of all types during the nominal 3 year mission lifetime.

To reach a $4 \mathrm{keV}$ low-energy threshold, the ECLAIRs detection plane is paved with $64004 \times 4 \mathrm{~mm}^{2}$ and 1 mm-thick Schottky CdTe detectors. The detectors are grouped by 32, in $8 \times 4$ matrices read by a low-noise ASIC, forming elementary modules called XRDPIX. In this paper, we also present our current efforts to investigate the performance of these modules with their front-end electronics when illuminated by charged particles and/or photons using radioactive sources. All measurements are made in different instrument configurations in vacuum and with a nominal in-flight detector temperature of $-20^{\circ} \mathrm{C}$. This work will enable us to choose the in-flight configuration that will make the best compromise between the science performance and the in-flight operability of ECLAIRs. We will show some highlights of this work.
\end{abstract}

Keywords: Gamma-ray bursts, X-/Gamma-rays, space mission, SVOM, astrophysics, instrumentation

\section{THE SVOM MISSION}

\subsection{Catching the most powerful transient events in the Universe}

Amongst the most powerful transient events known, Gamma-ray bursts (GRBs) appear to be the most extreme ones with isotropic radiated emission up to $\sim 10^{54} \mathrm{erg}$. They are highly transient and powerful cosmological explosions appearing randomly in the sky and characterized by very short (from a few milli-seconds to hundreds of seconds) prompt X-/Gamma-ray flashes followed by a multi-wavelength long-lasting afterglow emission (from days to months in some cases). They are often divided into two categories depending on their duration: short GRBs with duration typically less than $2 \mathrm{~s}$ and long GRBs with duration typically larger than $10 \mathrm{~s}$ (e.g. 1]), even if this classification could sometimes be misleading (e.g. 2 ). They are thought to be associated with the catastrophic formation of stellar mass black holes coupled with the launch of ultra-relativistic jets when some

Further author information: (Send correspondence to O. Godet)

Olivier Godet: E-mail: ogodet@irap.omp.eu, Telephone: 33561557536 
massive stars with masses larger than $10 M_{\odot}$ collapse (for long GRBs e.g. [3]) or when two neutron stars merge (e.g. [4]) for short GRBs.

GRB prompt and afterglow emission convey broadband information on the physics of the GRB phenomenon itself (e.g. particle acceleration in the jet and the radiation mechanisms), but also on the nature of the GRB progenitors with the detection of supernovae associated with long GRBs (e.g. [5, 6]) and the recent detection of kilonovae associated with some short GRBs (e.g. 7, 8]). Progress on these topics has been made possible with the launch of the Swift mission 9] in 2004, in particular on the afterglow and the nature of the progenitors of short GRBs (e.g. 7, 10 14]) as well as the characterization of the early afterglow (see e.g. [15 20]). However, there are still some puzzling questions that have to be answered on the prompt and afterglow emission as summarized in [e.g. 2, 20,21]. To be able to answer them, well characterized (spectrally and temporally) multiwavelength datasets of both prompt and afterglow emission of GRBs with measured redshifts are needed from future GRB missions along with some essential pieces of information to be found from particle and gravitational wave messengers. The detection of shock breakouts associated with normal SNe [e.g. 22 and low-luminosity GRBs [e.g. 6] offers a unique way to probe the connection between GRBs and classical supernovae, and how the core of a massive star collapses.

GRBs also convey a lot of excitement in cosmology and fundamental physics (e.g. ultra high-energy cosmic rays, high energy neutrinos and gravitational waves). Indeed, being very bright and located at cosmological distances - the farthest known GRB has a redshift of 8.2 ([23, see also [24] with a photometric redshift at 9.4 measured for GRB 090429B] - they pinpoint their host galaxy offering a way to study the first galaxies and maybe the first stars of the Universe, the so-called population III stars. They could be used to put constraints on a poorly known phase, the so-called re-ionization phase, when the matter of the Universe is thought to have been re-ionized by UV/X-ray photons from the first structures (stars and quasars), to track the evolution of baryons with redshift by studying the foreground material illuminated along their line of sight. If some properties of GRBs can be standardized as it is done for type-Ia supernovae, GRBs will offer a unique way to study the expansion of the Universe beyond redshift 2. Coalescence of two neutron stars thought to be the progenitors of some short GRBs are ideal sources to detect gravitational waves. Therefore, short GRBs offer an ideal way to pinpoint such systems on the sky for ground-based GW facilities such as aLIGO/aVirgo 25. The recent and future detections of kilo-novae associated with short GRBs also offer a way to study the nucleosynthesis of r-process elements and how they enrich the interstellar/intergalactic medium [e.g. 7, 8] .

The newly discovered class of ultra-long GRBs lasting several hundreds of minutes [e.g. 26 27] might indicate the existence of either a new class of star progenitor with much larger radii (e.g. blue giants) or another type of objects as the disruption of a white dwarf by an intermediate mass $\mathrm{BH}$ (IMBH - 27 29]). If it is the case, this offers very exciting prospects to detect strong GW signals [e.g. 30] and the long sought IMBH class that might be the building bricks to form supermassive black holes ( $\mathrm{SMBH}$ ) and that stay rather elusive (the only strong IMBH candidate being the ultra-luminous X-ray source HLX-1 in ESO 243-49 - 31]).

Tidal Disruption Events (TDEs - 32) are also an interesting class of energetic transient events as Swift showed by recently catching in real time a star being disrupted by a supermassive BH [33. TDEs offer the possibility to probe normally quiet supermassive $\mathrm{BHs}$ and hence to better constrain the fraction of occupation of BHs in the core of galaxies. The number of detected TDEs is likely to strongly increase in future years with monitoring of the transient sky in optical with the Large Synoptic Survey Telescope (LSST) and in radio with LOFAR (Low-Frequency Array for radio astronomy) and SKA (Square Kilometre Array).

Given this exciting and very promising science context for the future, the Chinese National Space Agency and the French Space Agency (CNES) have decided to jointly implement a mini-satellite mission dedicated to the study of Gamma-ray bursts and other powerful transient events for a launch around 2020. The mission SVOM (Space borne Variable Object Monitor) will operate à la Swift i.e. a platform consisting of a multi-wavelength payload coupled to rapid autonomous slewing capability [34- see Mercier et al. - paper 9144-73 this conference]. The science payload will consist of 2 wide-field cameras: the GRB trigger coded-mask camera ECLAIRs 35] and the Gamma-Ray Monitor [36] (see Wu et al.: paper 9144-195 this conference) as well as two narrow-field instruments, the Micro X-ray channel plate Telescope (MXT - [37]; see also Götz et al. paper 9144-74 this conference) working in the $0.3-10 \mathrm{keV}$ band and the Visible Telescope (VT - 38,39]) covering the B \& $\mathrm{R}$ visible bands. The development of the ECLAIRs and MXT instruments is led by the CNES in collaboration with several 


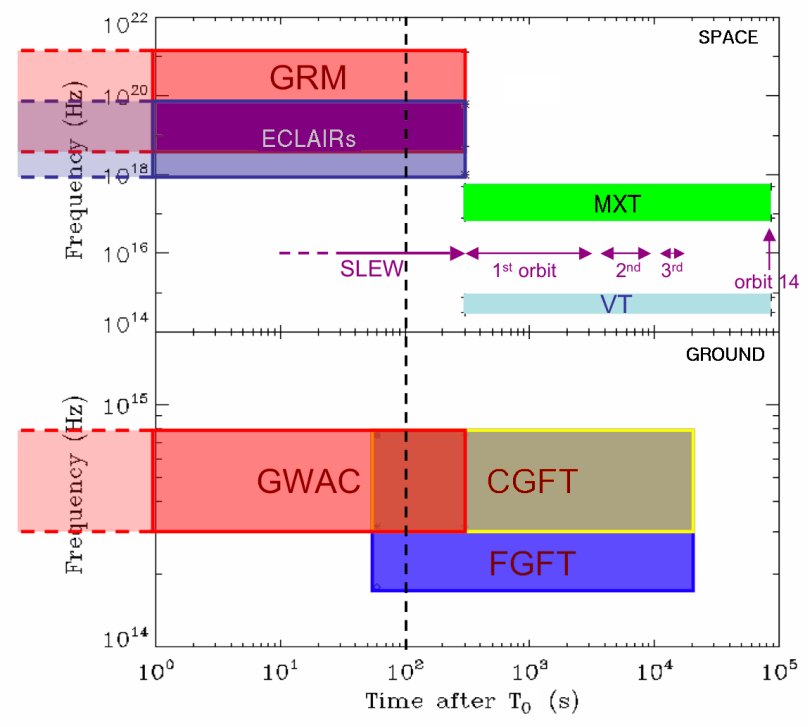

Figure 1. Broadband coverage from the space and ground instruments of SVOM as a function of time after the ECLAIRs GRB trigger $T_{0}$.

French and European astrophysics institutes. The IRAP is Principal Investigator of ECLAIRs, while the CEA is PI of the MXT. In addition to the space payload, SVOM will have a dedicated network of robotic narrow-field Ground Follow-up Telescopes (GFT) and Ground Wide Angle optical (V band) Cameras (GWAC) placed along the SVOM orbit in China and in Mexico. The French GFT (PI: IRAP) will have near infrared capability (J \& $\mathrm{H}$ bands) and maybe low resolution spectroscopy capability in addition to the use of the B, V, R, I filters, while the Chinese GFT will cover optical wavelengths. The GWAC camera led by China will be able to monitor a fraction of the ECLAIRs field of view (FoV) in order to search for optical prompt emission as observed for the naked eyed burst GRB 080319B [40] and other optical transients. Figure 1 gives an overview of the SVOM capability in terms of its temporal response as well as its spectral coverage from ground to space.

\subsection{Observing strategy}

SVOM will be operated in a Low Earth Orbit with an altitude of $625-650 \mathrm{~km}$ and an inclination around $30^{\circ}$. In order to facilitate the observations of SVOM GRBs by the largest ground based telescopes, and hence to increase the fraction of GRBs with measured redshift to be larger than 50\% (against nearly a third for the Swift mission from October 2004 to June 2014 ${ }^{*}$, SVOM will implement a nearly anti-solar pointing strategy with general avoidance of the Galactic center, Sco X-1 and the Moon in the ECLAIRs FoV in order not to reduce too much the ECLAIRs sensitivity to GRBs (see Fig. 2). In addition, for GRBs detected in the Galactic plane it will be harder to search for an optical counterpart due to dust extinction in the Galactic plane.

SVOM will be operated à la Swift in order to catch GRBs: ECLAIRs will scan specific regions of the sky compliant with the spacecraft attitude law (see Fig. 2) and the GRM will observe the same FoV. For $\sim 20 \%$ of the detected GRBs, the GWAC will be able to search for optical prompt emission on the ground. At $t=T_{0}$, ECLAIRS triggers on a burst, computes a position, sends it to the ground in near real time (less than 1 minute) via a network of VHF stations distributed along the SVOM orbit and requests a slew to the spacecraft in order to repoint the satellite so that the ECLAIRs GRB position is brought in the FoV of the MXT and VT in less than 5 min after $T_{0}$. Once possible, one of the two GFTs on the ground will start to observe the ECLAIRs error circle $1 \mathrm{~min}$ after $T_{0}$ in order to detect an optical afterglow counterpart and then to refine the positions. Each

\footnotetext{
*The Swift-BAT has detected 878 GRBs until June 2014, of which 278 GRBs had a measured redshift.
} 


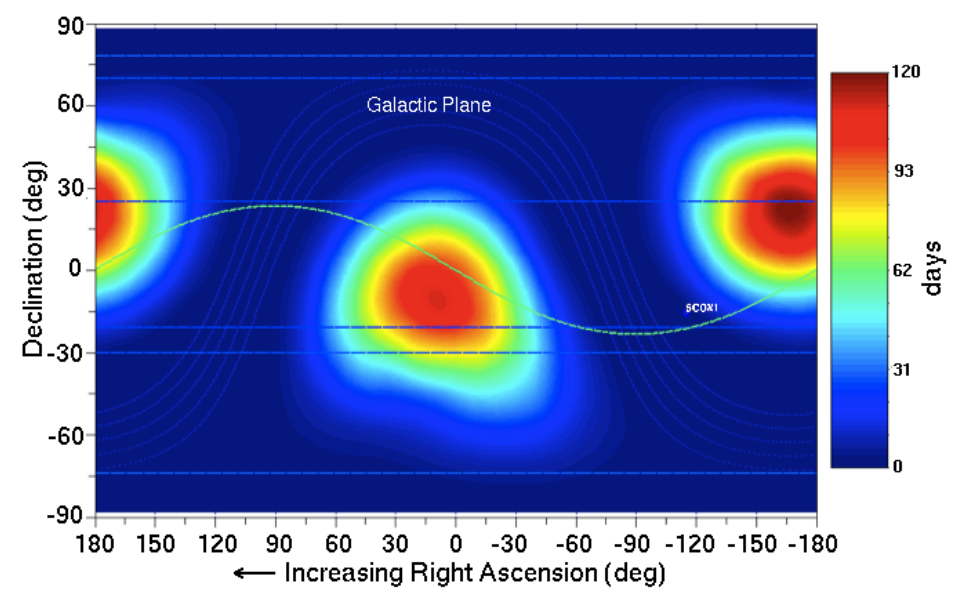

Figure 2. Sky map showing the pointing directions of the GRB trigger camera ECLAIRs. The SVOM orbit will be nearly anti-Sun and will avoid having the Galactic plane \& Sco X-1 in the field of view of ECLAIRs. The pointing color scale corresponds to the number of days during which a sky position will be in the field of view of ECLAIRs.

time a refined position is found by any of the SVOM instruments, an alert message will be promptly distributed to the world community.

\subsection{Mission requirements}

SVOM was conceived as a mini-satellite mission enabling further understanding of the GRB phenomenon and its related physics (and other energetic transient events) by providing a well characterized (spectrally and temporally) multi-wavelength dataset. This led us to define the science requirements listed below:

1. To enable the detection of all known types of GRBs, and particularly high-z GRBs for cosmological studies and low-z, sub-luminous GRBs to study in detail the connection with supernovae;

2. To quickly identify the rapidly decaying flux afterglows in X-rays and in optical;

3. To provide fast, reliable and accurate GRB positions (with accuracy less than 1 arcminute in X-rays and sub-arsec in optical) in order to maximize the probability of measuring a redshift and to perform cosmological studies with the large on-ground facilities;

4. To measure the temporal properties and the broadband spectral shape of the prompt emission (from visible to $\mathrm{MeV}$ ) in order to measure all the spectral parameters of the prompt emission, in particular the $E_{\text {peak }}$ parameter;

5. To quickly provide some GRB positions and parameters to encourage follow-up observations.

To implement such mission requirements has a profound impact on the design of the multi-wavelength payload as well as on the choice of the mission observing strategy as discussed in Section 1.2. In this paper, we focus on the GRB trigger camera ECLAIRs. First, we present the expected ECLAIRs performance (Section 2 to show that SVOM will be a strong contributor in the GRB field after 2020 and will also offer a good instrument sensitivity from optical to $\mathrm{keV}$ to perform non GRB science (Section 2.3). In Section 3, we present how the mission requirements are broken down into technical specifications for the design of the ECLAIRs camera, in particular for the detection plane. We will also describe our effort to characterize the performance of the elementary detector matrices that will pave the ECLAIRs detection plane (XRDPIX) and show some highlights of this work in Section 4. Section 5 is devoted to the conclusion. 

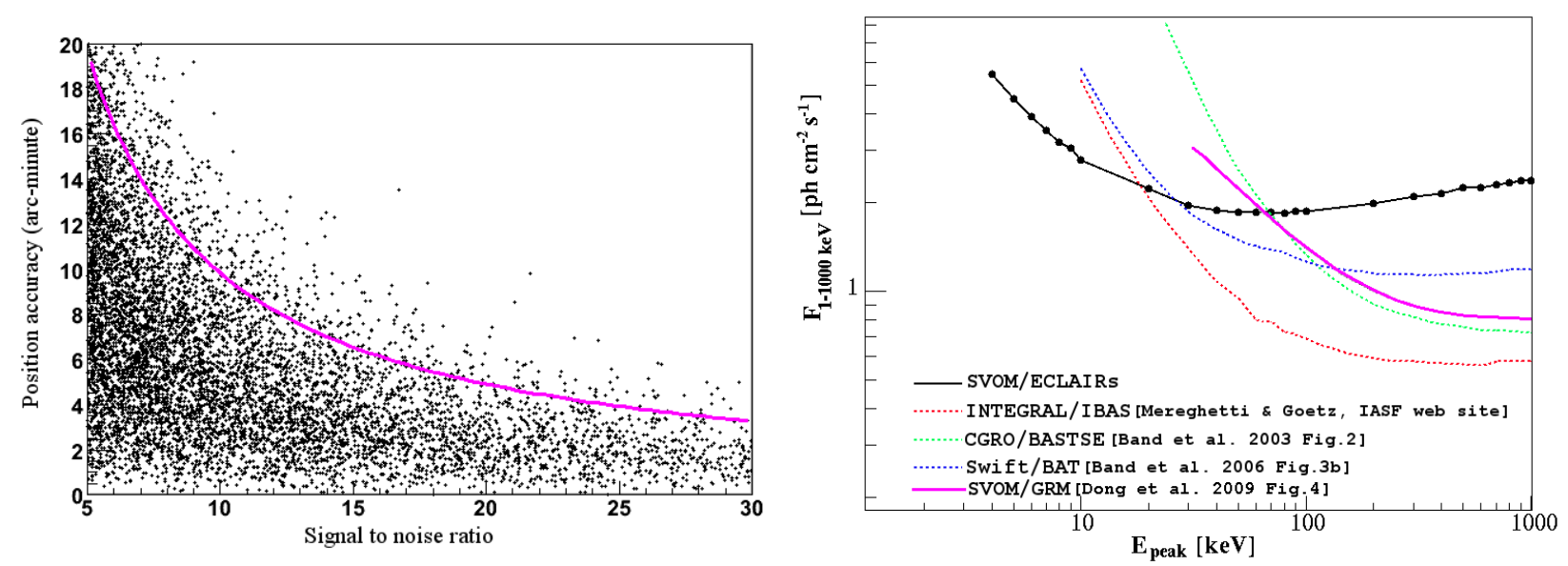

Figure 3. Left - Estimation of the ECLAIRs localization accuracy as a function of the estimated GRB signal-to-noise. We simulated $10^{5}$ GRBs with various properties within the ECLAIRs FoV in order to compute the GRB position accuracy using the ECLAIRs trigger algorithm simulator. The solid curve corresponds to the $90 \%$ confidence level error radius. Right - Simulated 1-1000 keV on-axis peak sensitivity of the GRB trigger camera ECLAIRs compared to other instruments as a function of the $E_{\text {peak }}$ parameter. We assumed a signal-to-noise ratio of $5.5 \sigma$, a GRB Band function with $\alpha=-1 \&$ $\beta=-3$ and an ECLAIRs imaging band from 4 to $50 \mathrm{keV}$. The SVOM/GRM sensitivity comes from 36].

\section{SCIENCE PERFORMANCE}

In this Section, we give an overview of the main expected performance of the ECLAIRs camera for GRB and non-GRB science.

\subsection{GRB localization accuracy}

Given the design constraints on ECLAIRs (see Section 3), a work of optimization of the mask pattern has been made with several objectives: i) to define a mask pattern with acceptable thermo-mechanical stresses and hence avoiding too large deformations that will introduce systematics in the image reconstruction; ii) to have a reasonably good positioning accuracy to localize GRBs (and other transient events) as well as to maximize the GRB sensitivity of ECLAIRs (Gros et al. in preparation). To do so, we studied the performance of masks with quasi-random patterns with different $\frac{m}{d}$ ratios with $m$ and $d$ being the linear size of a smallest mask hole and a CdTe pixel on the detection plane, respectively. From this work, the current baseline mask with $\frac{m}{d}=2.6$ is expected to provide a positioning accuracy of 13.9 arcminutes $(90 \%$ confidence level) for faint GRBs (the lower detection SNR threshold is currently set at 7) and a few arcminutes for bright ones (see the left panel in Fig 3). This value does not include the systematic errors. This mask also provides a gain in the GRB detection sensitivity of the order of $30 \%$ when compared to a mask with an initially (or previously) proposed value of $\frac{m}{d}=1.2$. The design of the MXT \& VT FoVs took into account the ECLAIRs positioning accuracy for faint GRBs in order to ensure the necessary refinement of the primary GRB position to a few dozens of arc-seconds with the MXT [see Götz et al. - paper 9144-74 this conference] and sub-arcsecond accuracy with the VT.

\subsection{Sensitivity to GRBs}

Fig. 3 (right panel) compares the $5.5 \sigma$ peak sensitivity for an on-axis GRB computed for ECLAIRs with other wide-field instruments: Swift-BAT, INTEGRAL/IBAS, CGRO/BATSE \& SVOM/GRM as a function of the GRB hardness. From this Figure, it is clear that ECLAIRs, thanks to a low energy threshold of $4 \mathrm{keV}$, will be as sensitive as the Swift-BAT for GRBs with $E_{\text {peak }}$-values less than $20 \mathrm{keV}$ (i.e. X-ray Flashes and potential high redshift GRBs).

Using the fraction of observed sky per year (that includes the ECLAIRs FoV, the Earth and SAA crossing), the expected level of the background in the ECLAIRs energy range [e.g. 41] and the $\log N-\log S$ function for long GRBs from [42], ECLAIRs is expected to be able to detect at least $\sim 180$ long GRBs over the 3 yr lifetime 

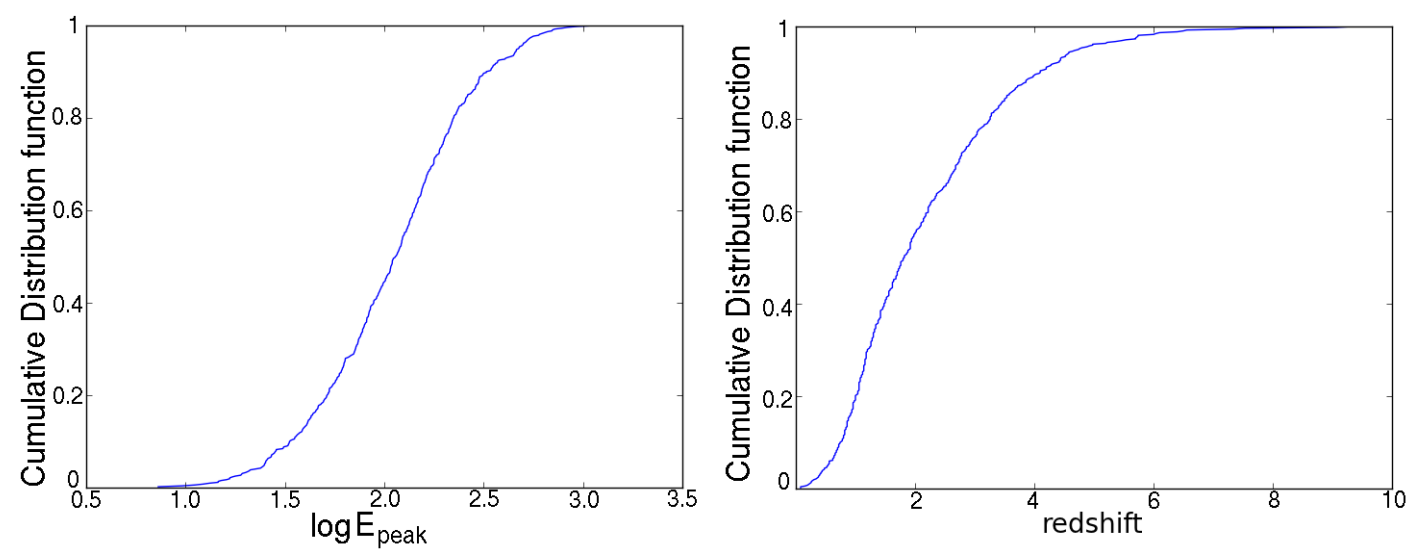

Figure 4. Simulated cumulative distribution function of GRBs detected by ECLAIRs as a function of $E_{p e a k}(l e f t)$ and redshift (right).

of the mission. We assumed here that a GRB is detected by ECLAIRs when the signal-to-noise ratio is more than 6. At least $10 \%$ should be added to take the short GRB population into account, giving a total of $\sim 200$ GRBs. An intensive work has been realized in order to refine this estimate using the ECLAIRs GRB trigger simulator, a physically-based synthetic GRB population \& the estimated in-orbit instrument background and taking into account the constraints induced by the SVOM pointing strategy (See Section 1.2), in particular the times when the Earth limb is within the ECLAIRs FoV. This fine work finds similar estimate for the ECLAIRs GRB detection rate. Figure 4 shows some predictions for the properties of the GRBs detected by ECLAIRs as a function of redshift and GRB hardness. 4\% of ECLAIRs GRBs are expected to be high redshift GRBs with $z>5$; which corresponds to a detection rate of nearly 3 high-z GRBs per year. This rate is comparable to the one of Swift-BAT. Most of ECLAIRs GRBs are also predicted to have $E_{\text {peak }}$ values less than $500 \mathrm{keV}$, indicating that for most SVOM GRBs it will be possible to measure the spectral parameters of the prompt emission using ECLAIRs and GRM data (see also Fig. 3).

\subsection{Non GRB science}

As for previous GRB missions, the SVOM observing time dedicated to GRB science will only represent a small fraction of the total observing time. In order to maximize the mission science return and to take advantage of the broad band coverage of the SVOM science payload, the SVOM consortium will implement a general non-GRB science program that will consists of: Target of Opportunities (ToOs) for unique objects \& a Guest Observer program opened to the whole community via a contact with one of the SVOM co-investigators, as well as key programs (e.g. monitoring of transient objects within the Galactic Center; follow-ups of some of the LSST, LOFAR, SKA or CTA transient events). Discussions about the key programs are still on-going within the SVOM consortium. The time devoted to the non-GRB science program is also expected to increase during the mission lifetime.

Here we outline potential targets that could be observed with ECLAIRs given the SVOM pointing strategy and the instrument sensitivity ( $\sim 50 \mathrm{mCrab}$ in an orbit in the $4-150 \mathrm{keV}$ energy band). For ECLAIRs, any sources located at $\pm 45^{\circ}$ from the pointing law could be observed (see Fig. 2). Figures 5 \& 6 shows an example of potential targets accessible to ECLAIRs.

\section{CURRENT DESIGN OF THE ECLAIRS GRB TRIGGER CAMERA}

The ECLAIRs GRB trigger camera [35] is based on the technology of coded mask cameras (e.g. 43]). ECLAIRs is developed by a consortium of French institutes (PI: IRAP - CEA-Saclay, IAP \& APC) under the supervision of the French Space Agency (CNES). Fig 7 shows the ECLAIRS sub-systems. Given the capability of the Chinese platform in term of mass and power available for the science payload, the design of the ECLAIRs instrument 


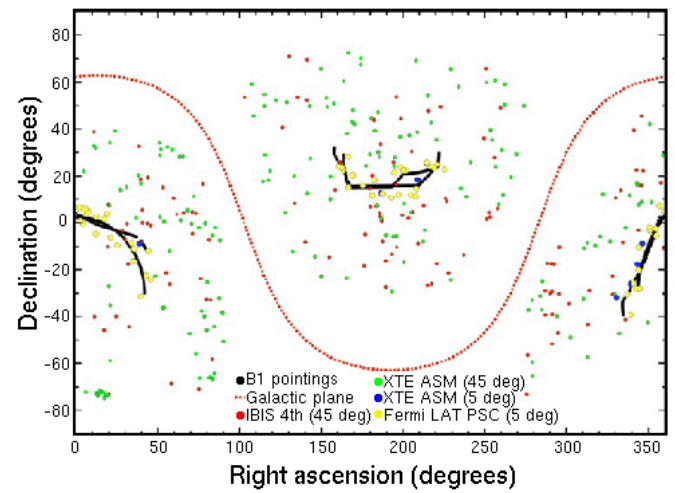

Figure 5. Sky map showing potential targets within the ECLAIRs FoV ( $\pm 45^{\circ}$ to the B1 law).
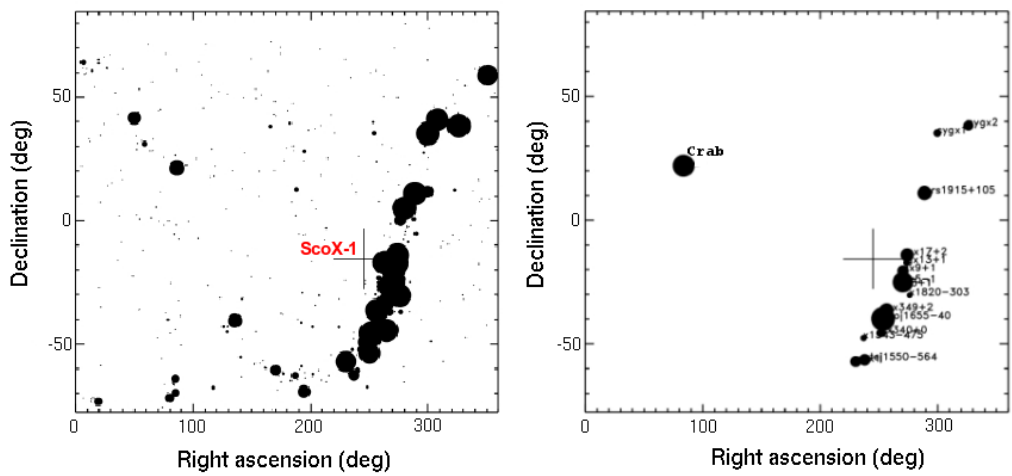

Figure 6. Left: The X-ray sky as seen by the RXTE All-Sky Monitor. Most of the X-ray sources are persistent. The size of the dots is proportional to the brightness of the X-ray sources. The cross indicates the location of Sco X-1. The sources outside the Galactic plane are mostly AGN. Right: The X-ray sky above 50 mCrab that roughly corresponds to the ECLAIRs sensitivity in just one orbit.

including the Data Process Unit should be compliant to the following mass and power allocation: $86.5 \mathrm{~kg}$ (with a $15 \%$ margin) and $84 \mathrm{~W}$ at most when working in the nominal configuration.

SVOM being a mini-satellite, only $\sim 1000 \mathrm{~cm}^{2}$ of geometrical surface could be allocated to the detection plane of ECLAIRs. To increase the sensitivity in particular to soft GRBs (X-ray Flashes and high redshift GRBs), it is then mandatory to have a low-energy threshold as low as possible (see Section 2.2). The goal is to have a scientific threshold of $4 \mathrm{keV}$ offering an effective area at this energy of $\sim 150 \mathrm{~cm}^{2}$. In order to reach this goal, we consider the use of $4 \times 4 \mathrm{~mm}^{2}$ and $1 \mathrm{~mm}$-thickness Schottky CdTe detectors which thanks to their Schottky anode and high resistivity offer low level of leakage current. The CdTe detectors will be operated below $-18^{\circ} \mathrm{C}$ (see Section 4). These detectors were provided by Acrorad Co., Ltd (Japan). Note that ohmic CdTe and CZT detectors were already used in space instrumentation (e.g. Swift/BAT [44 and INTEGRAL/ISGRI [45). However, the low-energy threshold of these instruments is larger than $10 \mathrm{keV}$. The flight detectors will be arranged over $8 \times 4$ detector matrices coupled with the CEA ASIC IDeF-X [46 [48], the so-called XRDPIX (see Fig. 7. [4] ), that will pave the detection plane (DPIX) making an array of $80 \times 80 \mathrm{CdTe}$ pixels mounted on a cold plate in AlBeMet. The IDeF-X ASIC shows a low intrinsic noise with an Equivalent Noise Charge floor of 33 electron r.m.s. for a peaking time of $6 \mu \mathrm{s}$, a low consumption $(3 \mathrm{~mW} /$ channel $)$ and is radiation hard (Single Event Latchup Linear Energy Transfer threshold of $56 \mathrm{MeV} \mathrm{cm}^{2} \mathrm{mg}^{-1}$ ). Given the 1-mm thickness of the detectors and the way the ASIC is working, the high energy threshold will be set at $150 \mathrm{keV}$. Therefore, the total energy range is expected to be from $4 \mathrm{keV}$ to $150 \mathrm{keV}$. The detection plane will be segmented into 8 sectors consisting of 25 XRDPIX (i.e. 800 CdTe detectors) to prevent any failure of the whole plane. Each sector will be connected to an electronic chain (ELS) that will work in the photon counting mode i.e. each event will be 

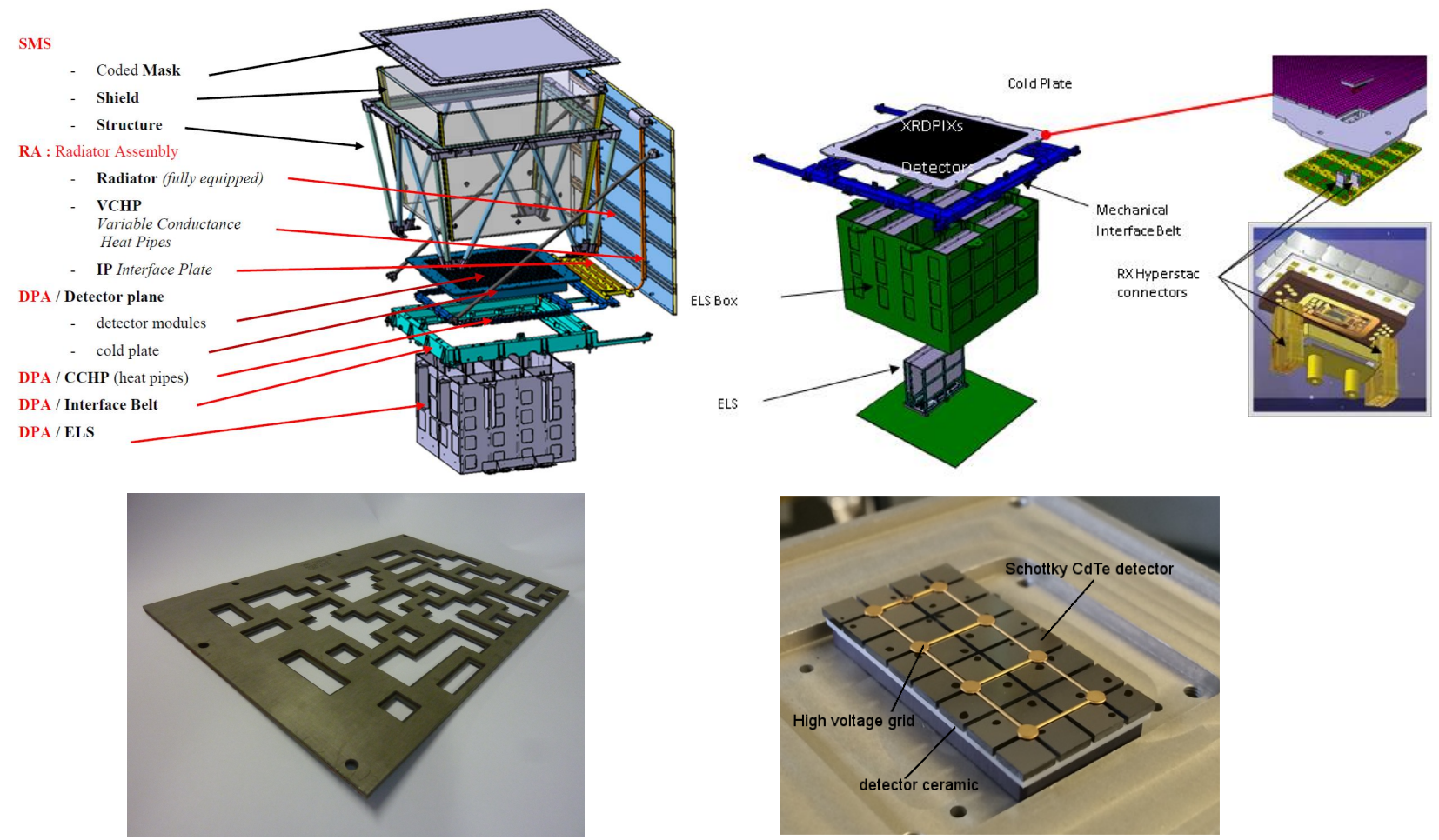

Figure 7. Top left: View of the coded mask camera ECLAIRs. The different sub-systems are shown, except the data processing unit in charge of the GRB detection and localization. Top right: View of the ECLAIRs detection plane (DPIX) paved with $80 \times 80$ Schottky CdTe detectors arranged into $8 \times 4$ detector matrices called XRDPIX. The detection plane is segmented into 8 sectors of 25 XRDPIX. Each sector is connected to an electronic chain (ELS) that will work in the photon counting mode i.e. each event will be time-tagged with a time resolution of $10 \mu \mathrm{s}$ and calibrated in energy in real time. The ELS will also perform pattern recognition flagging the events as single or multiple events. Bottom left: photo of a mock-up of the coded mask made of a sandwich of Ti-Ta-Ti. Bottom right: photo of an XRDPIX.

time-tagged with a time resolution of $10 \mu$ s and calibrated in energy in real time thanks to a FPGA. The ELS will also perform pattern recognition flagging the events as single or multiple events. The electronic chain is designed to support an event flow from an extremely bright GRB up to $10^{5}$ events $\mathrm{s}^{-1}$ over the all detection plane with a dead time of less than $5 \%$.

The calibrated events will be transmitted in real time via a space wire connection to the data process unit (UGTS - 50 ) that is in charge of detecting GRBs as well as managing the DPIX modes and instrument housekeeping. The UGTS will use the recorded events to reconstruct sky images every $20 \mathrm{~s}$ to search for the appearance of new sources within the ECLAIRs FoV by comparison with an on-board catalog of known X-ray sources. The UGTS will use both a count rate trigger algorithm on different timescales as well as an image trigger algorithm [51]. In case of a rate trigger, an automatic slew of the plate-form will only happen if it is confirmed by the appearance of a new source in the reconstructed images.

To maximize the GRB detection rate, a large FoV is required in order to be able to scan a large portion of the sky. Given the space limitations on the SVOM plate-form, ECLAIRs will have a large FoV of $89 \times 89 \mathrm{deg}^{2}$; which corresponds to $\sim 2$ steradian. The totally coded FoV is $22.1 \times 22.1 \mathrm{deg}^{2}$ (i.e. $\sim 0.15 \mathrm{sr}$ ). A graded passive shield combining $\mathrm{Pb}(0.9 \mathrm{~mm}), \mathrm{Cu}(0.1 \mathrm{~mm})$ and $\mathrm{Al}(0.5 \mathrm{~mm})$ will enclose the detection plane (DPIX) and the coded mask located at a distance of $46 \mathrm{~cm}$ from the DPIX (see Fig. 7). The choice of the material for the graded shield is a trade-off between a maximum reduction of the cosmic X-ray diffuse background in the 4-70 $\mathrm{keV}$ imaging band [41, mass budget considerations and the necessity to keep a few instrumental X-ray lines at higher energies for calibration purposes. 


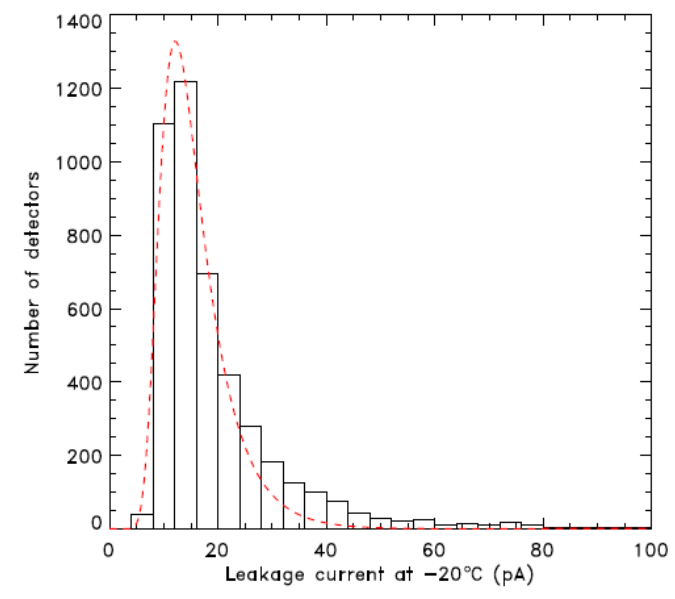

Figure 8. Distribution of the leakage current of the selected-to-the-flight CdTe detectors as measured at a temperature of $-20^{\circ} \mathrm{C}$ and a bias of $-600 \mathrm{~V}$. The figure comes from Remoue et al. (2010) 52. The median value is around $12 \mathrm{pA}$.

The mask will have a transparency of $40 \%$ (the mask transparency was initially of $30 \%$ and we changed it to $40 \%$ in order to improve the detection of short GRBs) and the blocking elements in the 4-70 keV band will be arranged into a quasi-random way. Given that the low energy threshold is expected to be at $4 \mathrm{keV}$, the use of a material supporting the blocking elements of the mask is not possible because any material at this energy will absorb a too important fraction of the incident photons, and hence this will reduce the effective area at low energy. Instead, the mask should be able to stand alone without any isolated blocking element. To do so, it will be pre-constrained before installed on the mechanical structure supporting the passive shield. Because of this, a special care has been made in the choice of the current baseline mask pattern to minimize the thermo-mechanical deformation of the mask (see also Section 2.1). The mask will be made of Ta (0.6 mm in thickness) sandwiched by two Ti sheets of $1 \mathrm{~mm}$ in thickness each in order to ensure the flatness of the mask (see Fig. 7). The mask will be covered by a multi-layer thermal coating insulation (MLI) to prevent optical photon loading on the detection plane.

\section{CHARACTERIZING THE PERFORMANCE OF THE XRDPIX}

The key science driver for the design of the detection plane of ECLAIRs is the low-energy threshold as mentioned in Section 3 (see also Section 2). In order to ensure that a low-energy threshold of $4 \mathrm{keV}$ could be reached, we defined a detailed test plan to validate the performance of each sub-system of the XRDPIX from the single CdTe detector to the complete XRDPIX by performing current, spectroscopic and noise measurements [e.g. 49.52 - see also Nasser et al.: paper 9144-150 this conference]. Several test facilities and software tools were then developed at the IRAP in order to perform these measurements (see Pons et al. - paper 9144-196 this conference).

\subsection{Selection of the Schottky CdTe detectors for the flight model}

An intensive work of leakage current and spectroscopic measurements on the all population of CdTe detectors purchased (12288 detectors) was realized at the IRAP in order to characterize the properties of Schottky CdTe detectors and to define selection criteria [52 that were used to select the 8000 flight-model (FM) detectors (i.e. 6400 detectors for the detection plane and 1600 detectors for spare modules). All the measurements were performed applying a reverse bias of $-600 \mathrm{~V}$ to the detectors and at two temperatures (the in-flight temperature of $-20^{\circ} \mathrm{C}$ and the ambient temperature of $+25^{\circ} \mathrm{C}$ ) in thermal controlled chambers. Note that the current measurements were made $30 \mathrm{~s}$ after biasing the detectors. Fig. 8 shows the distribution of leakage current measured at $-20^{\circ} \mathrm{C}$ for selected-to-the-flight detectors. The median value is around $12 \mathrm{pA}$. This value is well below the maximum value of the leakage current $(150 \mathrm{pA}-[52])$ required to reach a low energy threshold of 4 $\mathrm{keV}$ at the $6 \sigma$ level considering the total ASIC input capacitance of $10.5 \mathrm{pF}$ (worst case) and a peaking time of $6 \mu \mathrm{s}$. 


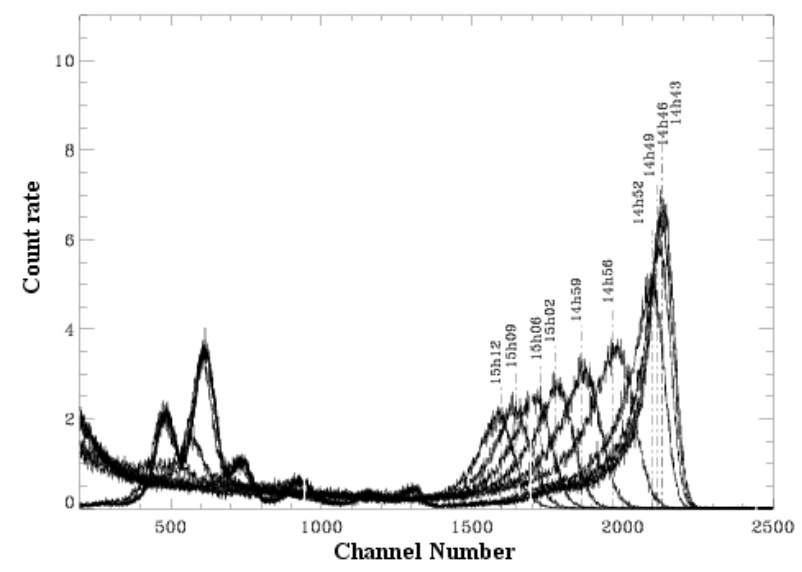

Figure 9. Degradation of the spectroscopic performance of a Schottky CdTe detector with time due to the polarization phenomenon. The spectrum was measured at $+25^{\circ} \mathrm{C}$ using a ${ }^{241}$ radioactive source. The detector was biased at $-100 \mathrm{~V}$.

The CdTe detectors considered for the detection plane being of Schottky type, these detectors are known to be unstable with time i.e. their leakage current increases over time due to the lowering of the Schottky potential height due to an accumulation of holes on the Schottky contact. We therefore performed current measurements over time $(\sim 2 \mathrm{~h})$ on a sample of more than 6000 detectors in order to characterize the temporal evolution of the leakage currents. We find that the increase in the leakage current over timescale of a day should be relatively moderate and this should not be considered as an issue regarding the ECLAIRs performance, in particular the low energy threshold 52].

The lowering of the Schottky potential height also induces a degradation of the spectroscopic performance of the detector with time. This is known as the polarization phenomenon 53,54. This degradation is characterized by a decrease in quantum efficiency (QE), an increase in spectral resolution (FWHM) and a shift of the photo-peak towards lower energies with time (see Fig. 9). This phenomenon is enhanced with the increase in temperature and the lowering of the reverse bias $[52-54]$. It starts immediately after the detectors have been biased. Initially the degradation in QE and FWHM and the shift of the photo-peaks are relatively small 52 ; Nasser et al.: paper 9144-150 this conference]. However, after the so-called polarization time $\left(t_{\text {pola }}\right)$, the spectroscopic degradation happens more rapidly. [52 measured a shift velocity of the ${ }^{241} \mathrm{Am} 59.6 \mathrm{keV}$ line of $V_{\text {shift }} \sim-64 \mathrm{eV} / \mathrm{min}$ before $t_{\text {pola }}$ and a polarization time of $t_{\text {pola }} \sim 11 \mathrm{~min}$ for a bias of $-100 \mathrm{~V}$ and a temperature of $+25^{\circ} \mathrm{C}$. Note that $t_{\text {pola }}$ and $V_{\text {shift }}$ increase rapidly with the temperature and the reverse bias. The regular depolarization of the Schottky CdTe detectors allows to restore the spectroscopic performance of the detectors. In orbit, it is expected to depolarize the detectors during deep passages of SVOM across the SAA (the duration of the longer passage outside the SAA is expected to be around 15 hours).

$\sim 76 \%$ of the all population of CdTe detectors purchased passed the selection criteria.

\subsection{Performance of the XRDPIX}

We focus here on our effort in characterizing in detail the performance of 5 proto-flight XRDPIX modules thanks to dedicated lab measurements that enable us to explore the behavior of these modules for various parameters (e.g. the reverse bias HT, the ASIC peaking time $t_{\text {peak }}$, the frequency of the FPGA clock). The main objectives of this work are to: i) improve our understanding of the physics behind Schottky type detectors (Nasser et al. - paper 9144-150 this conference; see also [52]); ii) study how the XRDPIX performance (e.g. the low-energy threshold, the quantum efficiency, the gain linearity, the polarization effect, etc.) evolve with these tunable parameters in order to establish a set of parameters to be used in-flight that will guarantee the stability of the instrument performance once in orbit. We also performed polarization measurements on several XRDPIX in order to ensure that for the in-flight configuration (see above) the impact of the polarization phenomenon on the detector spectroscopic performance could be almost neglected [see Nasser et al.: paper 9144-150 this conference]. 

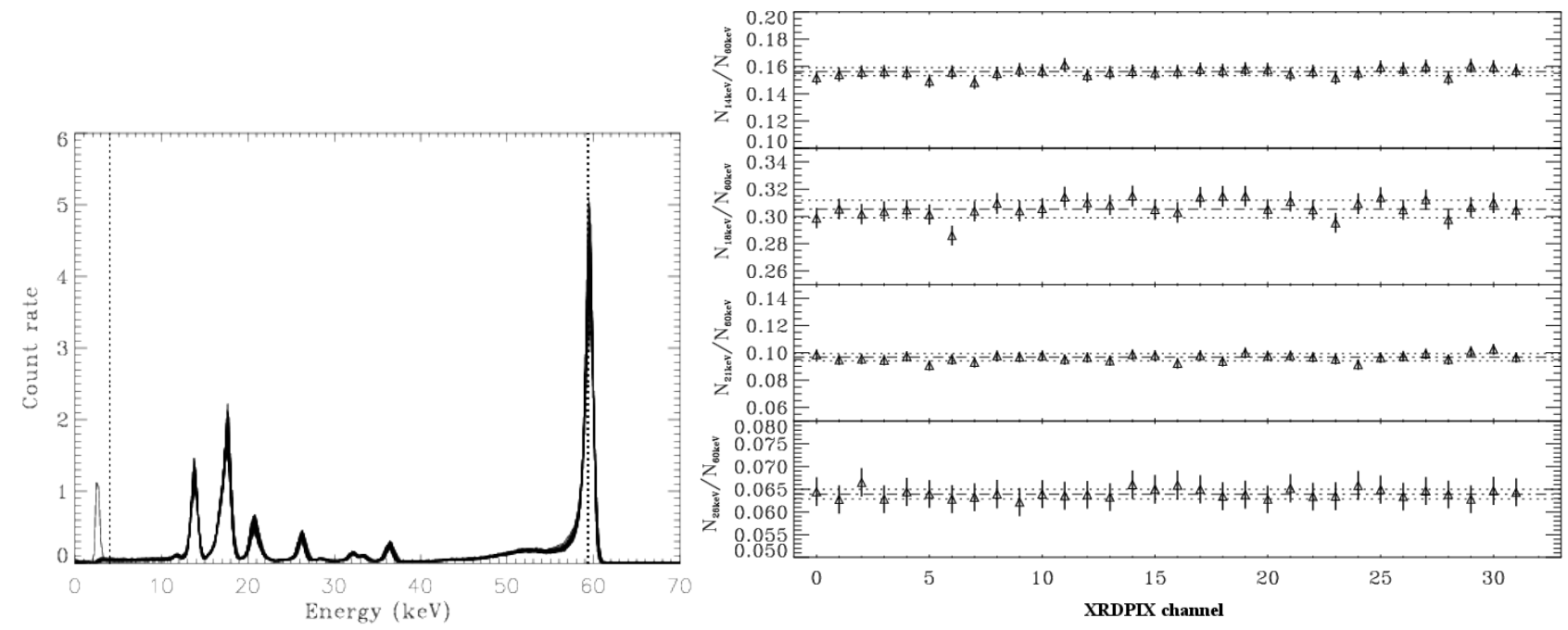

Figure 10. Left: Superposition of 32 spectra measured on one proto-flight XRDPIX (i.e. a 32 CdTe pixel matrix) obtained using a ${ }^{241} \mathrm{Am}$ radioactive source. The measurement was performed in the IRAP thermal-vacuum chamber at $T=-20^{\circ} \mathrm{C}$, a bias of $-400 \mathrm{~V}$ and a peaking time of $4.4 \mu \mathrm{s}$. Note that when the electronic noise peak is visible it is below 4 $\mathrm{keV}$ (the vertical dotted line). Right: Ratios of the number of counts under each ${ }^{241} \mathrm{Am}$ low energy lines over the number of counts under the ${ }^{241} \mathrm{Am} 59.6 \mathrm{keV}$ line for $32 \mathrm{CdTe}$ detectors of one XRDPIX. The measurements were also performed in the IRAP thermal-vacuum chamber at $T=-20^{\circ} \mathrm{C}$, a bias of $-400 \mathrm{~V}$ and a peaking time of $4.4 \mu \mathrm{s}$. The errors on the ratio-values are given at the $3 \sigma$ level. Note the very good homogeneity of the detectors.

A dedicated thermal-vacuum facility was built at the IRAP in order to realize all this work. The electronic chain used to perform these tests has the same functionalities as the in-flight chain. Most measurements were done in the photon counting mode and at the in-flight temperature of $-20^{\circ} \mathrm{C}$ using a ${ }^{241} \mathrm{Am}$ radioactive source.

We explore a large parameter space with 5 HT-values (from -250 to $-450 \mathrm{~V}$ ) and $7 t_{\text {peak-values (from } 0.95 \text { to }}$ $6.8 \mu \mathrm{s}$ ). We also considered the influence of adding an artificial dead-time of $34 \mu \mathrm{s}$ at the end of the ASIC event reading phase on the stability of the XRDPIX performance over time. The FPGA clock frequency was set at 4 $\mathrm{MHz}$ for all measurements. This work is still on-going.

We find from preliminary analysis of this work that the overall spectroscopic response of the CdTe detectors from different XRDPIX appears to be homogeneous within the different configurations considered as illustrated in Fig. 10 (see also Nasser et al. 2014 - paper 9144-150 this conference). The absence of the noise peak above 4 $\mathrm{keV}$ in most spectra (see the left panel in Fig. 10 indicates the very good performance of these Schottky CdTe detectors. During the advancement of this work, we also noticed that in rare cases a prominent "noise peak" could appear in some spectra (see Fig. 11). Note that the apparition of this prominent "noise peak" does not degrade too much the FWHM of the ${ }^{24} \mathrm{Am} 59.6 \mathrm{keV}$ line. Thus, we stress that this noise peak is unlikely to be related to a large increase in the level of the leakage current. Moreover, it appears that this "noise peak" varies with time. So, we suggest that this prominent "noise peak" may be due to the development of perturbations maybe related to the way the electronic chain works. Note that the electronic chain has not been fully validated yet. We are currently investigating the nature of these instabilities. We notice that the strength of this parasitic "noise peak" could be mitigated by decreasing the ASIC peaking time or by adding an artificial dead-time of $34 \mu \mathrm{s}$ at the end of the ASIC event reading phase.

\subsection{Performance of the electronic chain}

In addition to the measurements done at the IRAP, we also performed two test campaigns in 2010 and 2012 using the particle accelerator facility Tandem $\dagger^{\dagger}$ at the IPN Orsay in France. The goal was to simulate the in-flight

\footnotetext{
${ }^{\dagger}$ http://ipnweb.in2p3.fr/tandem-alto/tandem/
} 


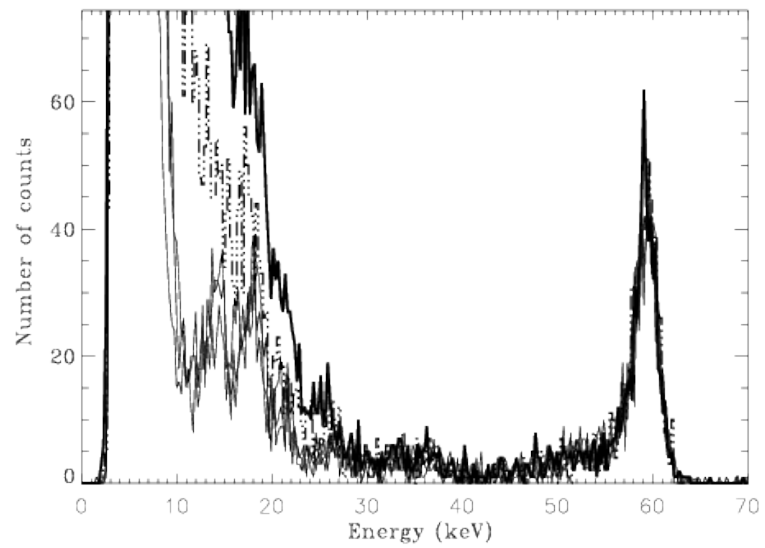

Figure 11. Superposition of ${ }^{241}$ Am spectra measured over successive epochs. The exposure time for each spectrum is $3 \mathrm{~min}$. The measurements were performed at $T=-20^{\circ} \mathrm{C}$, a bias of $-250 \mathrm{~V}$ and a peaking time of $6.8 \mu \mathrm{s}$. The thick dotted-dashed spectrum corresponds to the first measured spectrum, while the thick solid spectrum corresponds to the last measured spectrum. Note the evolution of the "noise peak" with time, while the spectral resolution of the ${ }^{241}$ Am $59.6 \mathrm{keV}$ line does not change significantly.

particle radiation environment of SVOM along its LEO orbit outside the SAA in order to study the impact on the ECLAIRs electronic chain: estimate the desaturation time and the impact on the energy coding of the photons [55. To do so, one XRDPIX operated under flight conditions (vacuum and $-20^{\circ} \mathrm{C}$ ) was irradiated with an incident flux of high energy $(20 \mathrm{MeV})$ protons and a ${ }^{241} \mathrm{Am}$ source. As illustrated in Fig. 12 for one XRDPIX pixel, the spectral response of the XRDPIX to photons is not degraded once it is irradiated with protons. The only difference from the spectra measured with and without the beam of protons on is the loss of some photon events due to the increase of the dead time in the former case. The level of dead time is estimated to be around $8.5 \%$. This experiment shows the robustness of the electronic chain to the particle flux irradiation expected on the SVOM orbit 55$]$.

\section{CONCLUSION}

With a launch expected around 2020, SVOM will be one of the main GRB missions (maybe the only one) combining regular alerts to the world community, a multi-wavelength coverage and rapid slewing capability. In addition, SVOM will work in a very exciting instrumental context with new ground and space facilities being operational such as the James Webb Space Telescope (mid and near IR) and the European-Extremely Large Telescope (near-IR/optical), Atacama Large Millimeter Array (sub-mm, mm), LSST (optical), SKA-pathfinder (radio) and Cherenkov Telescope Array at very high energy (TeV) on the ground. Such facilities with their very deep limiting sensitivity and observing strategy will open the era of multi-wavelength synoptic astronomy. This will be beneficial for studying the GRB afterglow emission over the broadband electromagnetic spectrum as well the environment of GRBs, their host galaxies in particular at high redshift. SVOM will also provide short GRB triggers to GW facilities such as aLIGO and aVirgo in order to search for GW signals from neutron star mergers. Synergy between SVOM and all these photonic and non-photonic facilities will enable to fulfill some interesting studies in cosmology and fundamental physics for GRB and non-GRB science.

In this paper, we presented the GRB trigger camera ECLAIRs on-board SVOM. We showed that the instrument performance will enable SVOM to detect a sample of at least 200 GRBs over the $3 \mathrm{yr}$ mission lifetime, including a sample of a few high-z GRBs and low-z sub-luminous GRBs. Fast distribution of the SVOM alerts (from ECLAIRs, MXT, VT \& GFTs) to the world community will enable to coordinate follow-up observations with ground and space facilities enabling GRB-based cosmological and fundamental physics studies. The performance of ECLAIRs are also expected to be adequate to observe a handful sample of other energetic transient events (e.g. TDEs, supernovae) that will also be observed at other wavelengths thanks to the MXT, VT and the 


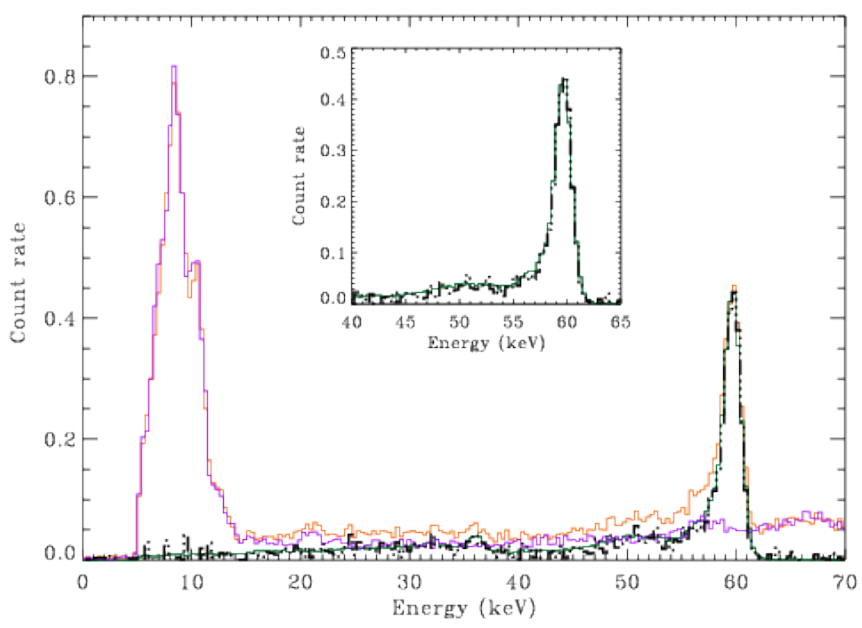

Figure 12. Spectra of one XRDPIX pixel as measured at the Tandem particle accelerator facility in order to investigate the impact of proton irradiation on the photon energy coding. The detectors were polarized at $-400 \mathrm{~V}$ and we used a peaking time of $4.4 \mu \mathrm{s}$. Green: the ${ }^{241} \mathrm{Am}$ spectrum with the proton beam off (REF). Only the ${ }^{241} \mathrm{Am} 59.6 \mathrm{keV}$ line is visible, because the other low energy lines were absorbed by the front entrance window of the thermal-vacuum chamber used during this experiment; Orange: the ${ }^{241}$ Am spectrum with the proton beam on (ALL); Blue: the spectrum induced by protons only (DARK). The thick dashed spectrum (RESTORE) corresponds to the contribution of the photons in the ALL spectrum (i.e. RESTORE $=$ ALL - DARK). The close-up shows a comparison of the reference ${ }^{241}$ Am spectrum (REF) and the RESTORE spectrum. We corrected the RESTORE spectrum for the effect of dead time (see the text). Note that the ${ }^{241} \mathrm{Am} 59.6 \mathrm{keV}$ line is not perturbed by the proton irradiation. The lines at low energies seen in the ALL and DARK spectra are fluorescence lines from the experimental setup materials induced by the proton irradiation.

ground instruments. We also stressed that ECLAIRs thanks to its sensitivity at low energy and its wide FoV will help following up transient events observed by other facilities in different wavelengths. We also presented the detailed characterization plan to ensure that the science requirements of the instrument could be reached, in particular the low energy threshold of $4 \mathrm{keV}$. From our lab measurements on characterizing the XRDPIX performance, we find that the preliminary spectroscopic results are very promising.

\section{REFERENCES}

[1] Kouveliotou, C. et al., "Identification of two classes of gamma-ray bursts," ApJ 413, 101 (1993).

[2] Zhang, B., "Gamma-ray bursts in the Swift era," Chinese Journal of Astronomy and Astrophysics 7, 1 (2007).

[3] MacFadyen, A. I. and Woosley, S. E., "Collapsars: Gamma-ray bursts and explosions in failed supernovae," ApJ 524, 262 (1999).

[4] Eichler, D. et al., "Nucleosynthesis, neutrino bursts and gamma-rays from coalescing neutron stars," $N a$ ture 340, 126 (1989).

[5] Galama, T. et al., "An unusual supernova in the error box of the $\gamma$-ray burst of 25 April 1998," Nature 395, 670 (1998).

[6] Campana, S. et al., "The association of GRB 060218 with a supernova and the evolution of the shock wave," Nature 442, 1008 (2006).

[7] Tanvir, N. et al., "A 'kilonova' associated with the short-duration -ray burst GRB 130603b," Nature 500, 547 (2013).

[8] E. Berger, W. F. . R. C., "An r-process kilonova associated with the short-hard GRB 130603b," ApJ 774, 23 (2013).

[9] Gehrels, N. et al., "The Swift gamma-ray burst mission," ApJ 611, 1005 (2004). 
[10] Gehrels, N. et al., "A short $\gamma$-ray burst apparently associated with an elliptical galaxy at redshift $z=0.225$," Nature 437, 851 (2005).

[11] Barthelmy, S. D. et al., "An origin for short $\gamma$-ray bursts unassociated with current star formation," $N a$ ture 438, 994 (2005).

[12] Church, R. P. et al., "Implications for the origin of short gamma-ray bursts from their observed positions around their host galaxies," MNRAS 413, 2004 (2011).

[13] Berger, E., "The environments of short-duration gamma-ray bursts and implications for their progenitors," New Astronomy Reviews 55, 1 (2011).

[14] Kopač, D. et al., "On the environment of short gamma-ray bursts," MNRAS in press, arXiv:1203.1864 (2012).

[15] Tagliaferri, G. et al., "An unexpectedly rapid decline in the X-ray afterglow emission of long $\gamma$-ray bursts," Nature 436, 985 (2005).

[16] Nousek, J. A. et al., "Evidence for a canonical gamma-ray burst afterglow light curve in the Swift XRT data," ApJ 642, 389 (2006).

[17] O'Brien, P. T. et al., "The early X-ray emission from GRBs," ApJ 647, 1213 (2005).

[18] Chincarini, G. et al., "The first survey of X-ray flares from Gamma-ray bursts observed by Swift: Temporal properties and morphology," ApJ 671, 1903 (2005).

[19] Kann, D. A. et al., "The afterglows of Swift-era gamma-ray bursts. i. comparing pre-Swift and Swift-era long/soft (type II) GRB optical afterglows," ApJ 720, 1513 (2010).

[20] Godet, O. and Mochkovitch, R., "Afterglows after Swift," Compte Rendu Physique 12, 276 (2011).

[21] Zhang, B., "Grb prompt emission: Open questions, debates, and a personal view," in [HIGH ENERGY GAMMA-RAY ASTRONOMY: 5th International Meeting on High Energy Gamma-Ray Astronomy], Proceedings, A. C., ed., 1505, 88 (2012).

[22] et al., A. M. S., "An extremely luminous x-ray outburst at the birth of a supernova," Nature 463, 469 (2008).

[23] Tanvir, N. R. et al., "A $\gamma$-ray burst at a redshift of z $\sim 8.2, "$ Nature 671, 1868 (2009).

[24] Cucchiara, A. et al., "A photometric redshift of z 9.4 for GRB 090429b," ApJ 736, 12 (2011).

[25] Corsi, A. et al., "Gravitational waves and gamma-ray bursts," in [Death of Massive Stars: Supernovae and Gamma-Ray Bursts], P. Roming, N. K. . E. P., ed., Proceedings IAU Symposium 279, arXiv:1204.4110 (2012).

[26] Gendre, B. et al., "The ultra-long gamma-ray burst 111209a: The collapse of a blue supergiant?," ApJ 766, 30 (2013).

[27] Levan, A. J. et al., "A new population of ultra-long duration gamma-ray bursts," ApJ 781, 22 (2014).

[28] Shcherbakov, R. V. et al., "GRB 060218 as a tidal disruption of a white dwarf by an intermediate-mass black hole," ApJ 769, 85 (2013).

[29] MacLeod, M. et al., "Illuminating massive black holes with white dwarfs: Orbital dynamics and high energy transients from tidal interactions," submitted to ApJ arXiv:1405.1426 (2014).

[30] Sesana, A. et al., "Observing white dwarfs orbiting massive black holes in the gravitational wave and electro-magnetic window," MNRAS 391, 718 (2008).

[31] Farrell, S. et al., "An intermediate-mass black hole of over 500 solar masses in the galaxy eso243-49," Nature 460, 73 (2009).

[32] Komossa, S., "Tidal disruption of stars by supermassive black holes: The x-ray view," in [Tidal Disruption Events and AGN Outbursts], Komossa, R. S. S., ed., EPJ Web of Conferences 39 (2012).

[33] Burrows, D. N. et al., "Relativistic jet activity from the tidal disruption of a star by a massive black hole," Nature 476, 421 (2011).

[34] Godet, O. et al., "The chinese-french svom mission: studying the brightest astronomical explosions," in [Space Telescopes and Instrumentation 2012: Ultraviolet to Gamma Ray], of the SPIE, P., ed., 8443, 10 (2012).

[35] Triou, H. E. et al., "The ECLAIRs telescope onboard the SVOM mission for gamma-ray burst studies," in [Hard X-Ray, Gamma-Ray, and Neutron Detector Physics XI], Ralph B. James, Larry A. Franks, A. B., ed., Proc. of SPIE 7749, 32 (2009). 
[36] Dong, N. R. et al., "The SVOM Gamma Ray Monitor," Science in China Series G: physics, Mechanics. E Astronomy 53, 40 (2009).

[37] Perinati, E. et al., "Leo radiation environment and the design of the micro-channel-plate x-ray telescope camera on-board the svom mission," in [Space Telescopes and Instrumentation 2012: Ultraviolet to Gamma Ray], of the SPIE, P., ed., 8443, 4 (2012).

[38] T. Wang, T., Qiu, Y., Cai, H., and Deng, J., "A fast onboard star-extraction algorithm optimized for the SVOM Visible Telescope.," Science in China Series G: physics, Mechanics. E3 Astronomy 53, 51 (2010).

[39] C. Wu, Y. L. Q. . H. B. C., "Svom visible telescope: Performance and data process scheme," in [Death of Massive Stars: Supernovae and Gamma-Ray Bursts], of the International Astronomical Union, P., ed., IAU Symposium 279, 421 (2012).

[40] Racusin, J. et al., "Broadband observations of the naked-eye big gamma-ray burst grb 080319b," Nature $\mathbf{4 5 5}$, $183(2008)$.

[41] Godet, O. et al., "Monte-carlos imulations of the background of the coded-mask camera for X- and gammarays on-board the Chinese-French GRB mission SVOM," NIM-A 603, 365 (2009).

[42] Butler, N. R. et al., "The cosmic rate, luminosity function, and intrinsic correlations of long gamma-ray bursts," ApJ 711, 495 (2010).

[43] Caroli, E. et al., "Coded aperture imaging in x- and gamma-ray astronomy," Space Science Reviews 45, 349 (1987).

[44] Barthelmy, S. et al., "The Burst Alert Telescope (BAT) on the SWIFT Midex mission," Space Science Reviews 120, 143 (2005).

[45] Lebrun, F. et al., "Isgri: The integral soft gamma-ray imager," A\&\&A 411, 141 (2003).

[46] Gevin, O. et al., "Idef-x eclairs: A cmos asic for the readout of cdte and cdznte detectors for high resolution spectroscopy," IEEE Transactions on Nuclear Science 56, 2351 (2009).

[47] Gevin, O. et al., "Idef-x v1.0: A new 16-channel low-noise analog front-end for cd(zn)te detectors," Nuclear Instruments and Methods in Physics Research A 567, 140 (2006).

[48] Gevin, O. et al., "IDeF-X ECLAIRs: An ultra low noise CMOS ASIC for the readout of Cd(Zn)Te detectors," in [Nuclear Science Symposium Conference Record], NSS, ed., Proc. of IEEE 1, 326 (2007).

[49] Lacombe, K. et al., "Development of a 32-detector cdte matrix for the svom eclairs x/gamma camera: Preliminary results," Nuclear Instruments and Methods in Physics Research A 732, 122 (2013).

[50] Provost, H. L. et al., "A scientific trigger unit for space-based real-time gamma ray burst detection (ii data processing model and benchmarks)," in [IEEE 2013 Nuclear Science Symposium], of IEEE, P., ed., 44, 7 (2013).

[51] Schanne, S. et al., "A scientific trigger unit for space-based real-time gamma ray burst detection (i - scientific software model and simulations)," in [IEEE 2013 Nuclear Science Symposium], of IEEE, P., ed., 1, 5 (2013).

[52] Remoué, N. et al., "Extensive testing of Schottky CdTe detectors for the ECLAIRs X-/gamma-ray camera onboard the SVOM mission," NIM-A 618, 199 (2010).

[53] Toyama, H. et al., "quantitative analysis of polarisation phenomena in cdte radiation detectors," Japanese Journal of Applied Physics 45, 8842 (2006).

[54] Cola, A. and Farella, I., "The polarization mechanism in cdte schottky detectors," Applied Physics Letters 94, 102113 (2009).

[55] Nasser, G. et al., "Impact of particles on the svom/eclairs x/gamma-ray camera electronic chain," to be submitted to NIM-A (2014). 